\title{
Time domain averaging and correlation-based improved spectrum sensing method for cognitive radio
}

\author{
Shenghong $\mathrm{Li}^{1 *}$ and Guoan $\mathrm{Bi}^{2}$
}

\begin{abstract}
Based on the combination of time domain averaging and correlation, we propose an effective time domain averaging and correlation-based spectrum sensing (TDA-C-SS) method used in very low signal-to-noise ratio (SNR) environments. With the assumption that the received signals from the primary users are deterministic, the proposed TDA-C-SS method processes the received samples by a time averaging operation to improve the SNR. Correlation operation is then performed with a correlation matrix to determine the existence of the primary signal in the received samples. The TDA-C-SS method does not need any prior information on the received samples and the associated noise power to achieve improved sensing performance. Simulation results are presented to show the effectiveness of the proposed TDA-C-SS method.
\end{abstract}

Keywords: Cognitive radio; Spectrum sensing; Time domain averaging; Correlation

\section{Introduction}

Cognitive radio (CR) networks allow unlicensed (or secondary) users to opportunistically exploit the underutilized spectrum bandwidth of the licensed (or primary) users. Spectrum sensing is a key operation performed by the CR networks to determine the spectrum holes of the spectrum allocated to a primary user. In the literature, several kinds of typical spectrum sensing methods have been reported, including energy detection methods $[1,2]$, matched filter detection methods [3,4], and cyclostationarity feature detection methods [5]. The energy detection methods need the prior knowledge of noise power and are vulnerable to the noise uncertainty. The matched filter detection methods need to know the waveform of the primary user in advance. The methods of cyclostationarity feature detection require the information on the cyclic frequencies of the primary user. The prior knowledge requirements of these methods often limit their realistic applicability. To avoid these undesirable requirements,

\footnotetext{
*Correspondence: shli@sjtu.edu.cn

1 Department of Electronic Engineering, Shanghai Jiao Tong University,

Dongchuan Road, Shanghai 200240, China

Full list of author information is available at the end of the article
}

some new statistical covariance-based methods for spectrum sensing in additive i.i.d white noise environments have been proposed [6-8]. These reported methods do not need the prior information about the signal and the noise power to achieve a sensing performance in a low signal-to-noise ratio (SNR) environment of $-22 \mathrm{~dB}$. However, these methods assume that the primary user's signal is a stationary random process and that all the received data samples contain the primary user's signal if it exists. In fact, most existing spectrum sensing methods require the last assumption. In fact, it is very possible that only a part of the received samples in practice contain the signal from the primary user or a part of or all the received samples are from some signals that are not stationary. For example, the initial transient signal, known as fingerprint of a wireless device [6], from the primary user is not stationary. If such samples are used, the methods reported in [7-9] will lose the supporting prerequisite and become unusable. Even in the cases that these required assumptions are valid, it is difficult for the methods in [7-9] to obtain the theoretical sensing performance in lower SNR environments when the number of the received samples available is limited.

\section{望 Springer}

(C) $2014 \mathrm{Li}$ and Bi; licensee Springer. This is an Open Access article distributed under the terms of the Creative Commons

Attribution License (http://creativecommons.org/licenses/by/2.0), which permits unrestricted use, distribution, and reproduction in any medium, provided the original work is properly credited. 
To overcome these problems and support more effective sensing in low SNR environments, we propose an effective time domain averaging and correlation-based spectrum sensing (TDA-C-SS) method based on time domain averaging and correlation for spectrum sensing in additive i.i.d white noise environments. Similar to the methods reported in [7-9], the proposed TDA-C-SS method does not need any aforementioned prior information and achieve desirable sensing performance in very low SNR environments. The TDA-C-SS method assumes the signal from the primary user to be deterministic. By making use of time domain averaging, the SNR of the received samples is increased. Then, the task of spectrum sensing is obtained by performing the correlation operation. Simulation results from various environments are presented to show the effectiveness of the proposed TDA-C-SS method.

The rest of this paper is organized as follows. Section 2 describes the system model and sampling operations to obtain the input sample sequence. Section 3 gives the proposed TDA-C-SS spectrum sensing method. Simulation results and discussions are presented in Section 4. Finally, conclusion is drawn in Section 5.

\section{System model and sampling description}

In a duration, $\left[t_{1}, t_{2}\right]$, of spectrum sensing, let $z(t)$ be the continuous-time signal received by the secondary user. The process of the spectrum sensing is regarded as a twohypotheses-test problem at a time duration, $\left[t_{1}, t_{2}\right]$, i.e., $\mathcal{H}_{0}$ : the primary signal is absent and $\mathcal{H}_{1}$ : the primary signal is present, described by

$$
z_{c}(t)= \begin{cases}w_{c}(t) & t \in\left[t_{1}, t_{2}\right] \mathcal{H}_{0} \\ s_{c}(t)+w_{c}(t) & t \in\left[t_{1}, t_{2}\right] \mathcal{H}_{1}\end{cases}
$$

where for $t_{1} \leq t_{3}, t_{4} \leq t_{2}$

$$
s_{c}(t)= \begin{cases}s_{\mathrm{pri}-\mathrm{c}}(t) & t \in\left[t_{3}, t_{4}\right] \\ 0 & \text { mul }\end{cases}
$$

and $s_{\text {pri-c }}(t)$ is any received primary signal, and $w_{c}(t)$ is the i.i.d white noise with a zero mean and a variance represented by $\delta^{2}$.

Let us be interested in the frequency band with central frequency $f_{0}$ and a narrow bandwidth $W$. The signal $z_{c}(t)$ defined in (1) is sampled at a frequency $f_{s} \leq L_{\text {mul }}\left(f_{0}+\right.$ $W / 2$ ), where, $L_{\mathrm{mul}}$ is a large constant, for example, $L_{\mathrm{mul}}=$
100. The choice of such values of $f_{s}$ and $L_{\text {mul }}$ is for the purpose of time domain averaging in our proposed method, which is to be described in Section 3. The two hypotheses in (1) are then expressed as

$$
z(n)= \begin{cases}w(n) & n=\left[1, \ldots, N_{0}\right] \mathcal{H}_{0} \\ s(n)+w(n) & n=\left[1, \ldots, N_{0}\right] \mathcal{H}_{1}\end{cases}
$$

where, $z(n)=z_{c}\left(n / f_{s}\right), s(n)=s_{c}\left(n / f_{s}\right)$ and $w(n)=$ $w_{c}\left(n / f_{s}\right)$.

It is worth noting that if $f_{0}+W / 2$ is very high, it is difficult to implement the sampling process because the available high speed samplers can support up to several tens of $\mathrm{GHz}$ [10-12]. Therefore, we should suitably select the value of $f_{0}+W / 2$ to allow an appropriate large value of $L_{\text {mul }}$ possible. It is also possible to use down conversion process [12] to generate the received samples when the value of $f_{0}+W / 2$ is very high.

\section{The proposed spectrum sensing method}

The proposed TDA-C-SS method treats the samples of the primary users to be deterministic. It firstly averages the received data samples in the time domain to increase SNR (or reduce the noise) then obtains the spectrum sensing by means of correlation operations.

\subsection{Sample time domain averaging}

Time domain averaging is an effective method to decrease noise power for periodic signal detection [13] (and therein [13]). For the obtained data sample sequence $z(n), n \in\left[1, N_{0}\right]$, the time average operation is defined by

$$
e(n)=\frac{\sum_{j=-M}^{M} z\left(n \cdot L_{\mathrm{mul}}+j\right)}{2 M+1} n=1, \ldots, N
$$

where $M$ is a small positive integer, for example, $M=5$, and $N=\left[N_{0} / L_{\text {mul }}\right]$.

If the samples from the primary users are treated as deterministic ones, $f_{s}$ is $L_{\text {mul }}$ times of $f_{0}+W / 2$ and $M$ is small, it means that

$$
s\left(n \cdot L_{\mathrm{mul}}\right) \approx \frac{\sum_{j=-M}^{M} s\left(n \cdot L_{\mathrm{mul}}+j\right)}{2 M+1}, n=1, \ldots, N
$$

with the assumption that $w_{c}(t)$ is i.i.d white noise, we easily obtain the following from (2):

$$
e(n)= \begin{cases}\frac{\sum_{j=-M}^{M} w\left(n \cdot L_{\mathrm{mul}}+j\right)}{2 M+1}=\omega(n) & \mathcal{H}_{0} \\ \frac{\sum_{j=-M}^{M} s\left(n \cdot L_{\mathrm{mul}}+j\right)}{2 M+1}+\frac{\sum_{j=-M}^{M} w\left(n \cdot L_{\mathrm{mul}}+j\right)}{2 M+1} \approx s\left(n \cdot L_{\mathrm{mul}}\right)+\omega(n) \mathcal{H}_{1}\end{cases}
$$


where $\omega(n)$ can be considered as the samples of the i.i.d white noise $\omega_{c}(t)$ with a zero mean and a variance of $\frac{\delta^{2}}{2 M+1}$. Let us define SNR $=10 \lg \frac{\sum_{n=1}^{N} s^{2}\left(n \cdot L_{\mathrm{mul}}\right)}{N \cdot \operatorname{Var}[\text { noise }]}$, where $\operatorname{Var}[$. denotes the variance of a random variable. It can be easily proved that the SNR of the sequence produced by the time averaging process is increased by $10 \lg (2 M+1) \mathrm{dB}$. If $M=5$, for example, the SNR of the time averaged output is increased by about $10 \mathrm{~dB}$. This gain in SNR is very valuable for effective spectrum sensing in the environment of strong noise.

\subsection{Correlation operation and sensing decision}

Similar to the method in [7], let us perform the correlation operation on the input samples and make a decision on the signal presence of the primary user based on a constructed correlation matrix. Being different from that in [7], however, the correlation used here is to be discussed from the view point of deterministic signal samples.

Let us consider the correlation defined by $r_{e}(i)=\frac{1}{N}$ $\sum_{n=1}^{N} e(n) e(n-i)$. Then, under $\mathcal{H}_{0}$, we have

$$
r_{e}(i)=\frac{1}{N} \sum_{n=1}^{N} \omega(n) \omega(n-i)
$$

Under $\mathcal{H}_{1}$, we have

$$
\begin{aligned}
r_{e}(i) \approx & \frac{1}{N} \sum_{n=1}^{N}\left[s\left(n L_{\text {mul }}\right)+\omega(n)\right]\left[s\left((n-i) L_{\text {mul }}\right)\right. \\
& +\omega(n-i)] \\
= & \frac{1}{N} \sum_{n=1}^{N} s\left(n L_{\text {mul }}\right) s\left((n-i) L_{\text {mul }}\right) \\
& +\frac{1}{N} \sum_{n=1}^{N} s\left(n L_{\text {mul }}\right) \omega(n-i) \\
& +\frac{1}{N} \sum_{n=1}^{N} s\left((n-i) L_{\text {mul }}\right) \omega(n) \\
& +\frac{1}{N} \sum_{n=1}^{N} \omega(n) \omega(n-i)
\end{aligned}
$$

It is noted that the samples from the i.i.d white noise, $\omega_{c}(t)$, which has a zero mean and a variance of $\frac{\delta^{2}}{2 M+1}$, the product of $s\left(n L_{\text {mul }}\right) \omega(n-i)$ are the samples from mutually independent random variables with a zero mean and a variance of $\frac{s^{2}\left(n L_{\mathrm{mul}}\right) \delta^{2}}{2 M+1}$. Therefore, based on the central limit theorem, it is concluded that the term $\frac{1}{N} \sum_{n=1}^{N} s\left(n L_{\text {mul }}\right) \omega(n-i)$ in (6) can be treated as a sample sequence of a Gaussian random variable $g$ $N\left(0, \frac{\delta^{2}}{(2 M+1) N^{2}} \sum_{n=1}^{N} s^{2}\left(n L_{\text {mul }}\right)\right)$ when $N \rightarrow \infty$. Because $\lim _{n \rightarrow \infty} \operatorname{Prob}|g-0|<\epsilon=1$ for an arbitrary positive $\epsilon$, we have $\frac{1}{N} \sum_{n=1}^{N} s\left(n L_{\text {mul }}\right) \omega(n-i) \rightarrow 0$ when $N \rightarrow \infty$. Similarly, we also conclude that the term $\frac{1}{N} \sum_{n=1}^{N} s((n-$ i) $\left.L_{\text {mul }}\right) \omega(n) \rightarrow 0$ in (6) when $N \rightarrow \infty$. In addition, according to statistics theory, it is also noted that when $N \rightarrow \infty,(5)$ or the last term in (6) satisfies

$$
\frac{1}{N} \sum_{n=1}^{N} \omega(n) \omega(n-i)=E\left[\omega_{c}(t) \omega_{c}(t-i)\right]= \begin{cases}\frac{\delta^{2}}{2 M+1} & i=0 \\ 0 & i \neq 0\end{cases}
$$

where $E[\cdot]$ is the expectation operation. Finally, the first term in (6), i.e. $\frac{1}{N} \sum_{n=1}^{N} s\left(n L_{\text {mul }}\right) s\left((n-i) L_{\text {mul }}\right), i=$ $1,2,3, \ldots$ are usually not identically zero when $s(n)$ is a deterministic sample sequence.

Let us construct an $L \times L$ correlation matrix in the same form as that in [6], i.e.,

$$
R_{e}=\left(\begin{array}{cccc}
r_{e}(0) & r_{e}(1) & \cdots & r_{e}(L-1) \\
r_{e}(1) & r_{e}(0) & \cdots & r_{e}(L-2) \\
\vdots & \vdots & \cdots & \vdots \\
r_{e}(L-1) & r_{e}(L-2) & \cdots & r_{e}(0)
\end{array}\right)
$$

Note that we have tested $P_{d}$ versus $L$ by experiments, and the experiment results show that when $L \geq 10, P d$ is not affected greatly by $L$, as a result, we choose the integer $L=10$ for the purpose of lower computation complexity. Based on the previous analysis of $r_{e}(i)$ values, apparently, the sensing test statistics and decision policies of the computer-assisted videodensitometry (CAV) method and the GCBA method in [7] are still applicable for our method. For the sake of simplicity, here, we use the test statistics and decision policy of the CAV method in [7], i.e., the test statistics is defined by [7]

$$
T=\frac{\sum_{n=1}^{L} \sum_{m=1}^{L}\left|r_{n m}\right|}{\sum_{m=1}^{L}\left|r_{m m}\right|}
$$

where $r_{n m}$ is the element located in the nth row and mth column of the matrix $R_{e}$. With a positive threshold value $\lambda$, the sensing decision for the primary signal is made by

$$
\begin{aligned}
& T>\lambda \text { Present } \\
& T \leq \lambda \text { Absent }
\end{aligned}
$$

\subsection{The TDA-C-SS Method}

Based on the discussion above, we propose the an improved spectrum sensing method as follows.

Step 1: sample the received signal at frequency $f_{s}$ to obtain the discrete samples $z(n), n=1,2, \ldots, N_{0}$. Step 2: for a given value of $M$, calculate $e(n), n=1,2, \ldots, N$ by (3).

Step 3: for a given value of $L$, calculate $r_{e}(i), i=0,1, \ldots, L-1$, and construct the matrix $R_{e}$ in (7).

Step 4: calculate $T$ in (8), and properly choose the value of $\lambda$ for the sensing decision.

Similar to the approach reported in $[7,9]$, let us use the computer simulation approach based on the given probability of false alarm, $P_{f}$, to choose the threshold $\lambda$. That is, first, a $P_{f}$ value is given and white noise is generated as the input, and then with a number of simulation results 
of $T$ in (8), the threshold value, $\lambda$, is selected to meet the requirement of $P_{f}$.

\subsection{Performance analysis of the proposed TDA-C-SS}

From the description above, it is seen that the proposed TDA-C-SS method does not need any prior information about the waveform and the cyclic frequencies of the primary user's signal and the noise power, which is similar to those methods in [7-9]. In contrast, our proposed method is also valid when only part of the received sample sequence contain the primary user's signal, which is the main different from those in [7-9] that assume that the primary user's signal is a stationary random process and that all the received samples must contain the primary user's signal. Therefore, the proposed method is more general and flexible.

By the averaging operation in the time domain, the proposed TDA-C-SS method is able to achieve an SNR improvement by $10 \lg (2 M+1) \mathrm{dB}$ compared with the CAV method in [7] for the same values of $N$ and $L$. Therefore, the proposed one is expected to improve the sensing performance substantially particularly in low SNR environments.

\section{Simulations}

In this section, simulation results are reported for the following three signal settings in the AWGN environments to verify the effectiveness of the proposed method.

Case I: the signal from the primary user is stationary. During spectrum sensing, all the received samples contain the signal of the primary user.

Case II: the signal from the primary user is stationary. During spectrum sensing, only a part of the received samples contain the signal of the primary user.

Case III: the signal from the primary user is not stationary.

For comparison, we also present the simulation results of the CAV method [6] applicable for case I. For all the simulations, the values of threshold $\lambda$ are chosen by the computer simulation approach described previously, and 1,000 Monte Carlo runs are carried out to estimate the value of $\lambda$.

\subsection{Simulation for case I}

We use a wireless microphone signal generated by the method in [14] with the following parameters: central frequency $f_{0}=100 \mathrm{MHz}$, bandwidth $W=36.8 \mathrm{KHz}$. Based on the discussion in Section 2, the sampling frequencies used for the proposed TDA-C-SS method and the CAV method are $f_{s}=10.3 \mathrm{GHz} \geq L_{\mathrm{mul}}\left(f_{0}+W / 2\right)$, where, $L_{\text {mul }}=100$, and $f_{s}=103 \mathrm{MHz} \geq f_{0}+W / 2$, respectively.

Figure 1 illustrates the detection probability $P_{d}$ versus SNRs with $P_{f}=0.1, L=10, N=50,000$. Figure 2 presents the detection probability $P_{d}$ versus the false

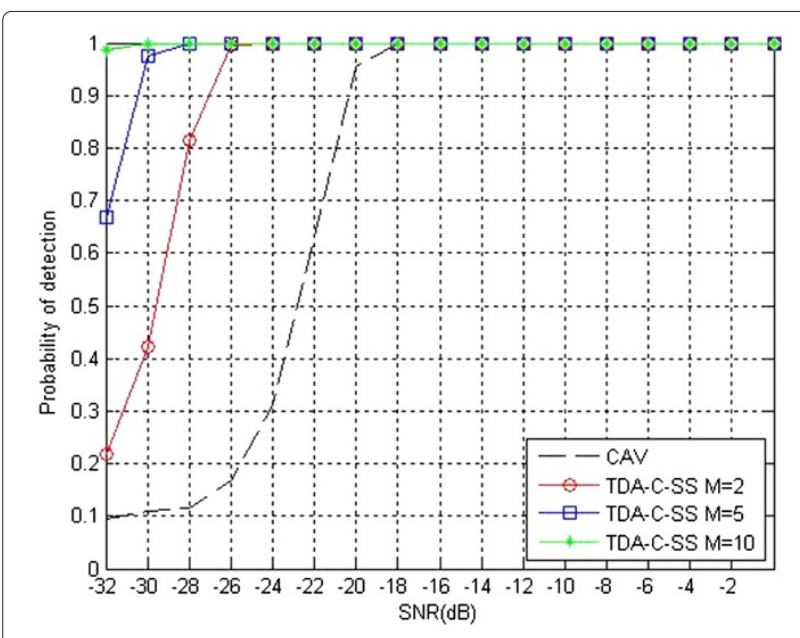

Figure $1 P_{d}$ curves versus SNR for case $I$.

alarm probability, $P_{f}$, with SNR $=-30 \mathrm{~dB}, L=10, N=$ 50,000 , for the proposed TDA-C-SS method, with different values of $M$, and the CAV method. From Figures 1 and 2, it is seen that under the same conditions, the detection performance of the proposed method is better than that of the CAV and can be improved further with increase of $M$. In addition, Figure 1 shows that, compared with the CAV method, the TDA-C-SS method achieves the improvement on the sensing performance by about $10 \lg (2 M+1) \mathrm{dB}$ in SNR. To achieve $P_{d}=0.985$, the values of the SNR needed by the CAV method and the TDA-CSS with $M=2,5$, and 10 are $-18.5,-26,-29.3$, and 32.2 $\mathrm{dB}$, respectively.

\subsection{Simulation for case II and case III}

In case II, the received sample sequence is 45,000 zero-valued signal samples followed by 5,000 wireless microphone signal samples that are generated in case I.

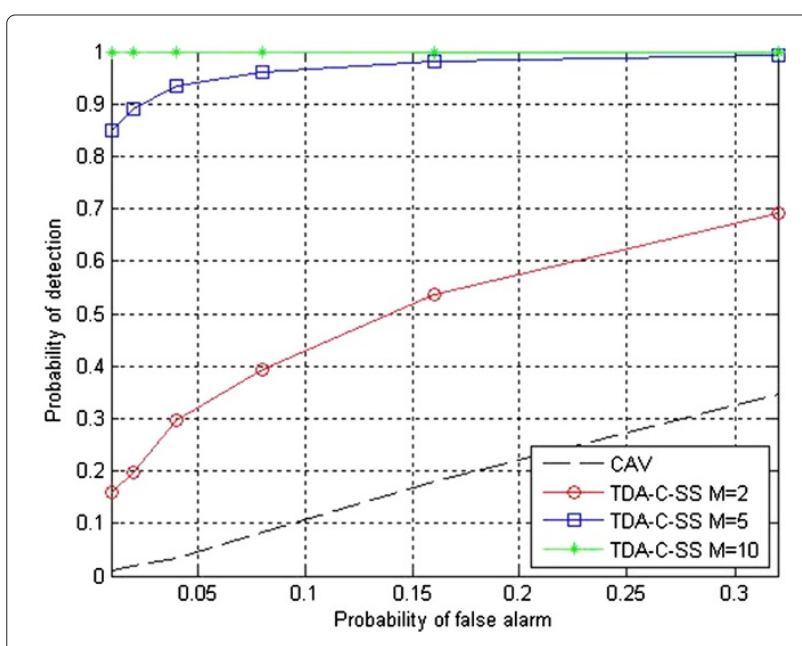

Figure $2 P_{d}$ curves versus $P_{f}$ for case $\mathrm{I}$. 
The segment of zero-valued signal samples is included for the frequently observed situation in which the primary user begins to occupy its channel after the secondary user has monitored the channel for certain period of time. The sampling frequency for the TDA-C-SS method is $f_{s}=10.3 \mathrm{GHz}$. For case III, we use a fingerprint signal, which is generated by using the unit-step response of a second-order RLC parallel circuit described by a differential equation:

$$
\frac{d^{2} i_{l}(t)}{d t^{2}}+2 \sigma \frac{d i_{l}(t)}{d t}+w_{0}^{2} i_{l}(t)=\frac{1}{L_{f} C_{f}} i_{s}(t)
$$

with $L_{f}=10^{-8}, C_{f}=10-10, R=0.08, \sigma=R / 2 L_{f}=$ $4,000,000, w_{0}=\sqrt{1 / L_{f} C_{f}}=10^{9}$. The fingerprint signal has the central frequency $f_{0}=160 \mathrm{MHz}$ and the bandwidth $W=8 \mathrm{MHz}$. The sampling frequency for the TDA-C-SS method is $f_{s}=16.4 \mathrm{GHz} \geq L_{\text {mul }}\left(f_{0}+W / 2\right)$, where, $L_{\text {mul }}=100$.

It is not reasonable to use the CAV method because the signals used in case II and case III are not stationary. Therefore, we only verify the effectiveness of the proposed TDA-C-SS method. Figures 3 and 4 give, respectively, detection probability versus SNRs for the two cases with $P_{f}=0.1, L=10, N=50,000$, and the detection probability $P_{d}$ versus the false alarm probability $P_{f}$ with $\mathrm{SNR}=-30 \mathrm{~dB}, L=10, N=50,000$. From these figures, it is seen that the TDA-C-SS method is still effective because this method does not consider the properties of the received signal. Similarly, the detection performance can be improved further with the increased value of $M$.

\section{Conclusion}

In this paper, an improved spectrum sensing method, TDA-C-SS, based on time domain averaging and correlation

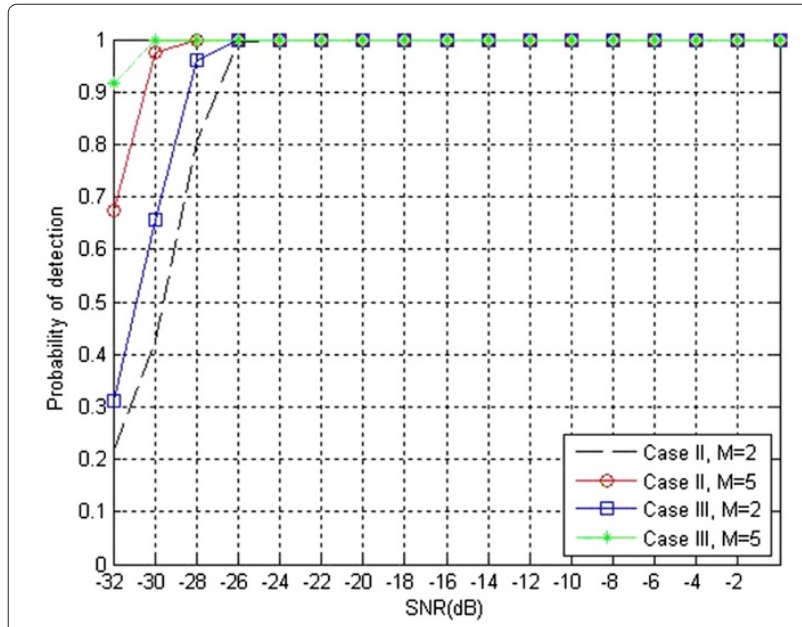

Figure $3 P_{d}$ versus SNR obtained by the TDA-C-SS method for case II and case III.

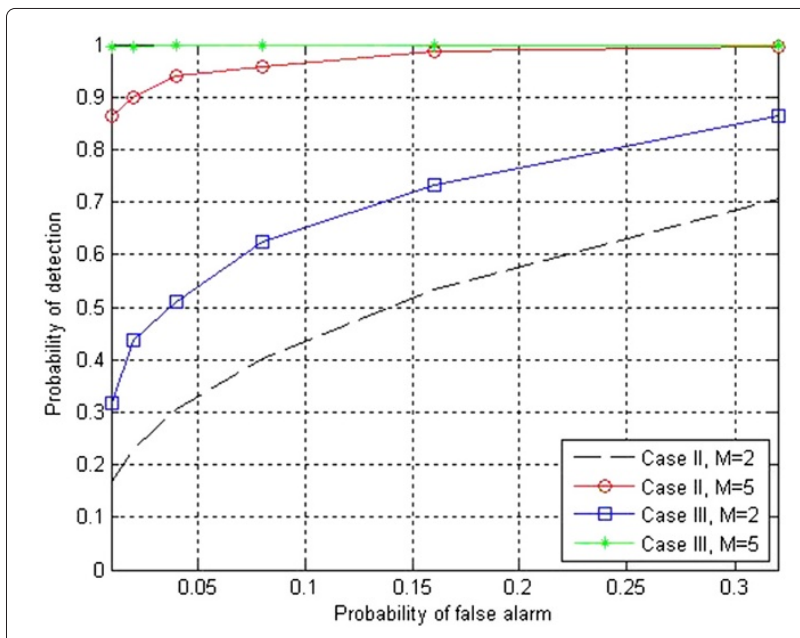

Figure $4 P_{d}$ curves versus $P_{f}$ of the TDA-C-SS for case II and case III.

has been proposed. The time domain averaging process has been typically used to decrease the noise effects, and correlation matrix has been constructed to decide the existence of the primary user's signal. In comparing with other reported method, such as CAV method, the proposed one can sense a primary user's signal in the white noise environment in very low SNR environments without requiring any prior knowledge about the signal and noise power. In particular, the proposed method is flexible to effectively sense the signals that are not stationary. Our simulation results have shown the desirable advantages of the proposed methods.

\section{Competing interests}

The authors declare that they have no competing interests.

\section{Acknowledgements}

This work is funded by the National Science Foundation of China (61271316, 61071152), 973 Program (2010CB731403, 2010CB731406, 2013CB329605) of China, Chinese National 'Twelfth Five-Year' Plan for Science \& Technology Support (2012BAH38 B04), Key Laboratory for Shanghai Integrated Information Security Management Technology Research, and Chinese National Engineering Laboratory for Information Content Analysis Technology.

\section{Author details}

${ }^{1}$ Department of Electronic Engineering, Shanghai Jiao Tong University, Dongchuan Road, Shanghai 200240, China. ${ }^{2}$ Information Engineering School of EEE, Nanyang Technological University, 50 Nanyang Ave, Singapore 639798, Singapore.

Received: 20 November 2013 Accepted: 16 January 2014 Published: 4 April 2014

\section{References}

1. D Cabric, SM Mishra, RW Brodersen, Implementation issues in spectrum sensing for cognitive radios, in Proc. Asilomar Conf. Signals, Syst. Computers (Pacific Grove, CA, USA, 2004), pp. 772-776

2. A Sahai, D Cabric, Spectrum sensing: fundamental limits and practical challenges, in IEEE Int. Symp. New Frontiers DySPAN (Baltmore, Maryland, USA, 2005), pp. 1-90

3. D Cabric, A Tkachenko, RW Brodersen, Spectrum sensing measurements of pilot, energy, and collaborative detection, in Proc. IEEE Military Commun. Conf. (MILCOM) (Washington, D.C., USA, 2007) 
4. HS Chen, W Gao, DG Daut, Signature based spectrum sensing algorithms for IEEE 802.22 WRAN, in Proc. IEEE Int. Conf. Communications (ICC) (Glasgow, Scotland, UK, 2007), pp. 6487-6492

5. N Han, SH Shon, JO Joo, JM Kim, Spectral correlation based signal detection method for spectrum sensing in IEEE 802.22 WRAN systems, in Proc. Intern. Conf. Advanced Commun. Technology (Phoenix Park, South Korea, 2006), pp. 1765-1770

6. JY Xu, HS Zhang, Detection to primary user based on radio frequency fingerprint in cognitive radio, in Proc. Intern. Cong. Image and Signal Processing (CISP) (Tianjin, China, 2009)

7. Y Zeng, YC Liang, Spectrum-sensing algorithms for cognitive radio based on statistical covariance. IEEE Trans. Veh. Technol. 58(4), 1804-1815 (2009)

8. Y Zeng, YC Liang, Eigenvalue-based spectrum sensing algorithms for cognitive radio. IEEE Trans. Commun. 57(6), 1784-1793 (2009)

9. Y Zeng, YC Liang, R Zhang, Blindly combined energy detection for spectrum sensing in cognitive radio. IEEE Signal Process. Lett. 15, 649-652 (2008)

10. T Lecklider, Raising the B/W bar to $32 \mathrm{GHz}$. Eval. Eng. 49(6), 34 (2010)

11. YJ Zhao, YH Hu, HJ Wang, Enhanced random equivalent sampling based on compressed sensing. IEEE Trans. on Instrum. Meas. 61(3), 579-586 (2012)

12. R Lopez-Valcarce, G Vazquez-Vilar, Wideband spectrum sensing in cognitive radio: joint estimation of noise variance and multiple signal levels, in IEEE International Workshop on Signal Processing Advances for Wireless Communications (SPAWC 2009) (Perugia, Italy, 2009), pp. 96-100

13. M Pini, DM Akos, Exploiting GNSS signal structure to enhance observability. IEEE Trans. Aerospace Electron. Sys. 43(4), 1553-1565 (2007)

14. Y Zeng, YC Liang, Performance of eigenvalue based sensing algorithms for detection of DTV and wireless microphone signals, in IEEE 802.22-06/186r0 (Phoenix Park, South Korea, 2006)

doi:10.1186/1687-6180-2014-46

Cite this article as: $\mathrm{Li}$ and Bi: Time domain averaging and correlation-based improved spectrum sensing method for cognitive radio. EURASIP Journal on Advances in Signal Processing 2014 2014:46.

\section{Submit your manuscript to a SpringerOpen ${ }^{\circ}$ journal and benefit from:}

- Convenient online submission

- Rigorous peer review

- Immediate publication on acceptance

- Open access: articles freely available online

- High visibility within the field

- Retaining the copyright to your article

Submit your next manuscript at $\boldsymbol{\wedge}$ springeropen.com 